\title{
A GROUP AHP CONSENSUS REACHING MODEL FOR SUPPLIER SELECTION IN COLLABORATIVE PRODUCT DEVELOPMENT
}

\author{
Qingxing Dong \\ School of Information Management \\ Central China Normal University \\ Wuhan, China \\ E-mail: qx_dong@126.com \\ Keyu Zhu \\ School of Management \\ Key Laboratory of Process Optimization and Intelligent Decision-making, Ministry of \\ Education \\ Hefei University of Technology \\ Hefei 230009, China \\ E-mail: cauchyandy@163.com
}

\begin{abstract}
Group consensus is an essential factor of a successful group decision. However, judgments are always diverse in the real world. Thus supporting the process of consensus reaching is of great significance. To improve the group consensus, the moderator of a group can give some recommendations to the incompatible decision makers to revise extreme opinion. Also, in an autocratic group, where the decision makers are the experts or consultants providing their suggestion to the leader or client, the moderator can adjust the weight or importance of the incompatible decision maker to reduce the perturbation from the extreme opinion. In this paper, we propose a consensus reaching model for the autocratic group decision, where the members use the Analytic Hierarchy Process (AHP) to express their judgment. In this dynamic and interactive model, a moderator suggests the incompatible expert to revise his/her judgment. If the expert rejects this suggestion, his/her importance weight will be adjusted downward. This process supports the leader or client to make a successful decision with a dispersed group of expert by improving the consensus level in this group. Finally, a numerical example is given to illustrate the validity of the proposed consensus reaching model.
\end{abstract}

Keywords: Analytic Hierarchy Process (AHP); Group AHP, Consensus reaching; Weight adjustment; Judgment updating

\section{Introduction}

To make a successful group decision, a certain level of consensus in a group must be achieved. Consensus is commonly meant as a total agreement of all the decision makers with respect to all judgments. However, the opinions in a group are always diverse. Thus 
measuring and improving the consensus level in a group is of great significance in group decision making.

\section{Literature Review}

There is considerable literature on improving group consensus. defined the degree of consensus and presented a consensus model based on linguistic preference. In a group AHP decision making context, proposed a framework to measure the group consensus level and then use such information to support the process of consensus building. proposed a consensus reaching model for group AHP under row geometric mean prioritization method. This model first defines the consensus indices among the PCMs and then the moderator suggests decision maker to adjust his/her PCM. presented a model to improve both the consistency and consensus in group AHP, in which the consensus was measured by the compatibility index of two PCMs and then the decision maker would revise his/her PCM according to the moderators suggestion.

The consensus models described above are focused on revising or updating the decision makers' judgments. The weights of the decision makers, however, which are usually associated with the quality of their judgments, are kept fixed in the negotiation and discussion process. It is well-known that in democratic group decision making (e.g. a presidential election, congressional vote), it is inadvisable and infeasible to change the weight of a decision maker simply because his opinion is incompatible with that of the group. But it is feasible when a group making decision in an autocratic environment, e.g., the decision about the date of D-Day, where the decision makers in this group are the experts or consultants who input their opinions to a powerful decision maker. Thus changing the importance weight vector of individuals in a group is an alternative way of encouraging them to reach a group consensus.

\section{Hypotheses/Objectives}

The consensus model presented in this paper attempts to update both the opinion and weights of the decision makers in a negotiation process. The weight adjustment method is simplified and easy to execute in each round of the process. In our model, a decision maker is able to either insist on using his/her own judgment or update his/her judgment based on the moderator's suggestion and his/her weight is adjusted automatically according to which choice he/she makes in the negotiation process.

\section{Research Design/Methodology}

For simplicity, we use $N=\{1,2, \mathrm{~L}, n\}, M=\{1,2, \mathrm{~L}, m\}$ to denote elements in sets. For a finite set of alternatives $X=\left\{x_{1}, x_{2}, \mathrm{~L}, x_{n}\right\}$, the judgment information is represented as an $n \times n \operatorname{PCM} \boldsymbol{A}=\left(a_{i j}=w_{i} / w_{j}\right)_{n \times n}$, where $a_{i j}=1 / a_{j i}$ and $a_{i j}$ belongs to Saaty's 19 fundamental scale and represents the relative importance or better, dominance of $x_{i}$ over $x_{j}$.We assume that there are $m$ decision makers $D M_{1}, D M_{2}, \mathrm{~L}, D M_{m}$ with PCM $\boldsymbol{A}_{k}=\left(a_{i j(k)}\right)_{n \times n}$, for $k \in M$, and let $\boldsymbol{\rho}=\left(\rho_{1}, \rho_{2}, \mathrm{~L}, \rho_{m}\right)^{\mathrm{T}}$ be the weight or importance vector of the decision makers, where $\rho_{k} \geq 0, \sum_{k=1}^{m} \rho_{k}=1, k \in M$. Then by aggregating with the weighted geometric mean, the group PCM $\boldsymbol{G}=\left(g_{i j}\right)_{n \times n}$ can be calculated as 


$$
g_{i j}=\prod_{k=1}^{m}\left(a_{i j(k)}\right)^{\rho_{k}}
$$

Since there is almost always a diversity of opinions, a consensus reaching process is needed to drive decision makers towards consensus. To measure the consensus level in a group, one first measures the closeness of two Pairwise Comparison Matrices(PCMs). Considering that PCM belongs to an absolute scale and thus also to a ratio scale, Saaty suggested that the closeness of two PCMs can be measured by using the compatibility index. The entire process of our consensus reaching model would be as shown in Fig. 1.

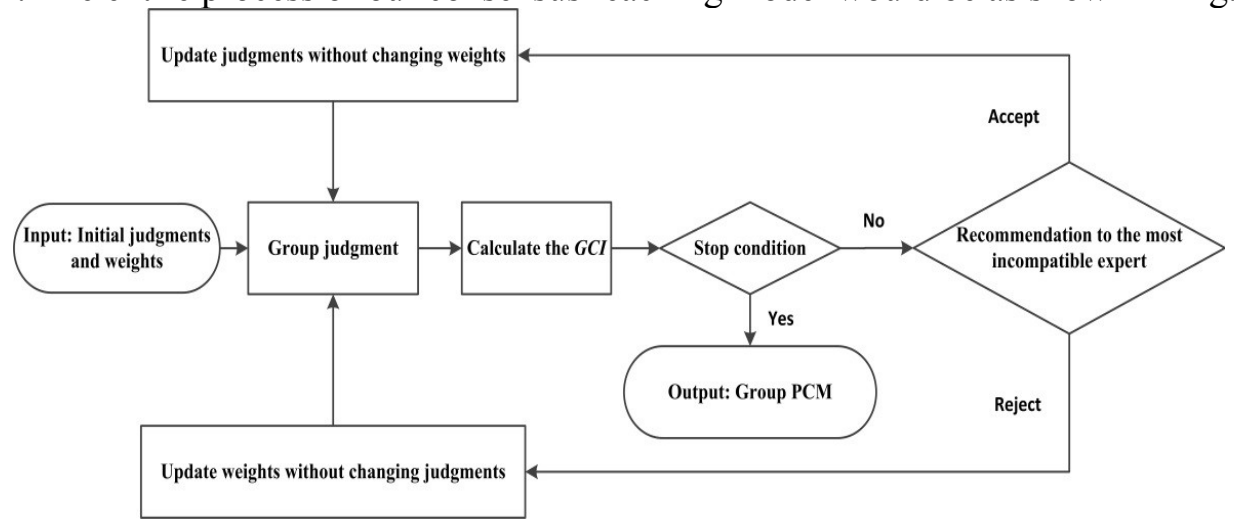

\section{Data/Model Analysis}

We use the following group decision making problem presented by to demonstrate the validity of our proposed process. Suppose we have four alternatives $X_{1}, X_{2}, X_{3}$ and $X_{4}$ to be ranked and five decision makers $D M_{1}, D M_{2}, D M_{3}, D M_{4}$, and $D M_{5}$ with PCMs $\boldsymbol{A}_{k}=\left(a_{i j(k)}\right)_{4 \times 4}, k=1,2,3,4,5$, where

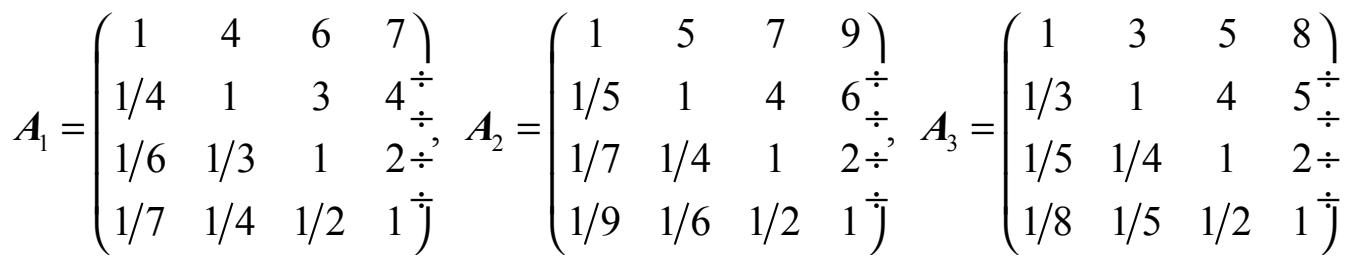

$$
\begin{aligned}
& A_{4}=\left(\begin{array}{cccc}
1 & 4 & 5 & 6 \\
1 / 4 & 1 & 3 & 3 \dot{\div} \\
1 / 5 & 1 / 3 & 1 & 2 \div \\
1 / 6 & 1 / 3 & 1 / 2 & 1
\end{array} A_{5}=\left(\begin{array}{cccc}
1 & 1 / 2 & 1 & 2 \\
2 & 1 & 2 & 3 \dot{\div} \\
1 & 1 / 2 & 1 & 4 \div \\
1 / 2 & 1 / 3 & 1 / 4 & 1
\end{array}\right)\right.
\end{aligned}
$$

The consistency ratios of $A_{k}$ are $C R_{A_{1}}=0.0383, C R_{A_{2}}=0.0678, C R_{A_{3}}=0.0339$, $C R_{A_{4}}=0.0471, C R_{A_{5}}=0.0363$, which indicate that the given PCMs are of acceptable consistency. Let $\boldsymbol{\rho}=(0.1,0.3,0.1,0.2,0.3)^{\mathrm{T}}$ be the initial weight vector of the decision makers. Set the threshold value of the group consensus index $\varepsilon=1.01, \alpha=0.7$, $\theta=0.7$, and the maximum number of iterations $T=10$. Then we show how to apply 
IJAHP Article: Mu, Saaty/A Style Guide for Paper Proposals To Be Submitted to the International Symposium of the Analytic Hierarchy Process 2014, Washington D.C., U.S.A.

the proposed consensus reaching model to adjust the weights and update the judgments. Let $t=0, \boldsymbol{A}_{k}^{0}=\boldsymbol{A}_{k}, k=1,2,3,4,5, \boldsymbol{\rho} \boldsymbol{\rho}=$. We get the group PCM

$$
\boldsymbol{G}^{0}=\left(\begin{array}{cccc}
1 & 1.9786 & 2.6547 & 3.3879 \\
0.5054 & 1 & 2.2894 & 2.8536 \\
0.3767 & 0.4368 & 1 & 1.7818 \div \\
0.2952 & 0.3504 & 0.5612 & 1
\end{array}\right)
$$

Then we can calculate $G C I_{k}^{0}, k=1,2,3,4,5$. The consensus reaching process should be continued until it fulfills the stop conditions. The group consensus index, weight vector and the choice of decision makers in each iteration are listed in Table 1.

Table 1. Group consensus index, weight vector and the choice of selected decision maker

$$
\begin{aligned}
& \text { Round }\left({ }^{t}\right) \text { Group consensus index }(\boldsymbol{G C I}) \text { and weight vector }(\lambda) \begin{array}{c}
\text { Selected } \\
\text { decision maker }
\end{array} \text { Choice } \\
& 0 \quad \boldsymbol{G} \boldsymbol{C} \boldsymbol{I}^{0}=(1.0502,1.1144,1.0382,1.0040, \mathbf{1 . 3 3 6 6})^{\mathrm{T}} \\
& \boldsymbol{\rho}^{0}=(0.1,0.3,0.1,0.2,0.3)^{\mathrm{T}} \quad D M_{5} \quad \text { Accept } \\
& 1 \\
& \boldsymbol{G C} \boldsymbol{I}^{1}=(1.0301,1.0817,1.0225,1.0253, \mathbf{1 . 2 1 7 7})^{\mathrm{T}} \\
& \boldsymbol{\rho}^{1}=(0.1,0.3,0.1,0.2,0.3)^{\mathrm{T}} \\
& D M_{5} \quad \text { Accept } \\
& \boldsymbol{G} \boldsymbol{C} \boldsymbol{I}^{2}=(1.0182,1.0603,1.0139,1.0175, \mathbf{1 . 1 3 1 7})^{\mathrm{T}} \\
& \boldsymbol{\rho}^{2}=(0.1,0.3,0.1,0.2,0.3)^{\mathrm{T}} \\
& \boldsymbol{G} \boldsymbol{C I ^ { 3 }}=(1.0112,1.0459,1.0094,1.0137, \mathbf{1 . 0 8 0 6})^{\mathrm{T}} \\
& \boldsymbol{\rho}^{3}=(0.1,0.3,0.1,0.2,0.3)^{\mathrm{T}} \\
& \boldsymbol{G C} \boldsymbol{I}^{4}=(1.0071,1.0361,1.0073,1.0122, \mathbf{1 . 0 4 9 7})^{\mathrm{T}} \quad D M_{5} \quad \text { Reject } \\
& 4 \\
& \boldsymbol{\rho}^{4}=(0.1,0.3,0.1,0.2,0.3)^{\mathrm{T}} \\
& \boldsymbol{G C I}^{5}=(1.0042, \mathbf{1 . 0 2 7 4}, 1.0065,1.0119,1.0626)^{\mathrm{T}} \\
& \boldsymbol{\rho}^{5}=(0.1225,0.3225,0.1225,0.2225,0.21)^{\mathrm{T}} \\
& \boldsymbol{G C} \boldsymbol{I}^{6}=(1.0052, \mathbf{1 . 0 1 7 3}, 1.0075,1.1005,1.0553)^{\mathrm{T}} \\
& \boldsymbol{\rho}^{6}=(0.1225,0.3225,0.1225,0.2225,0.21)^{\mathrm{T}} \\
& D M_{5} \quad \text { Accept } \\
& D M_{5} \quad \text { Accept } \\
& 5 \\
& 6 \\
& \boldsymbol{G C \boldsymbol { I } ^ { 7 }}=(1.0063,1.0110,1.0086,1.0098,1.0500)^{\mathrm{T}} \\
& \boldsymbol{\rho}^{7}=(0.1225,0.3225,0.1225,0.2225,0.21)^{\mathrm{T}} \\
& D M_{2} \quad \text { Accept } \\
& 7 \\
& \boldsymbol{G C I}^{8}=(1.0074,1.0070,1.0098,1.0094,1.0459)^{\mathrm{T}} \\
& \text { 8(Stop) } \\
& \boldsymbol{\rho}^{8}=(0.1225,0.3225,0.1225,0.2225,0.21)^{\mathrm{T}} \\
& D M_{2} \quad \text { Accept } \\
& D M_{2} \quad \text { Accept } \\
& D M_{2} \quad \text { Accept }
\end{aligned}
$$


As can be seen in Table 2, the algorithm terminates after 8 iterations. The final group $\mathrm{PCM}$ is

$$
\boldsymbol{G}^{*}=\left(\begin{array}{cccc}
1 & 3.2469 & 4.7410 & 6.3679 \\
0.3080 & 1 & 3.2353 & 4.0455 \div \\
0.2109 & 0.3091 & 1 & 2.1451 \div \\
0.1570 & 0.2472 & 0.4662 & 1
\end{array}\right)
$$

From $\boldsymbol{G}^{*}$ we can derive the final priorities $\boldsymbol{w}=(0.5691,0.2559,0.1094,0.0656)^{\mathrm{T}}$. Thus the ranking of alternatives should be $X_{1} \mathrm{f} X_{2} \mathrm{f} X_{3} \mathrm{f} X_{4}$.

\section{Limitations}

This model is feasible when a group making decision in an autocratic environment, where the decision makers in this group are the experts or consultants who provide their opinion to a powerful decision maker as the reference. It's not a democratic model.

\section{Conclusions}

In this paper, we have proposed a consensus reaching model for the autocratic group AHP. We presented a consensus reaching process for group AHP based on weight adjustment and judgment updating. The main advantages of this model are: (1) This model allows the involved decision makers to choose to accept or reject the recommendation from moderator; (2) The weight adjustment is used as an incentive for a decision maker to update judgment according to moderator's suggestion; (3) The consistency of PCMs is maintained in the proposed consensus reaching model; (4) The proposed consensus reaching model improves the consensus level of a group.

Additional research is currently underway to improve the accuracy and effectiveness of dealing with incompatibility.

\section{Key References}

Ben-Arieh, D., Zhifeng, C., 2006. Linguistic-labels aggregation and consensus measure for autocratic decision making using group recommendations. Systems, Man and Cybernetics, Part A: Systems and Humans, IEEE Transactions on 36, 558-568.

Bryson, N., 1996. Group decision-making and the analytic hierarchy process: Exploring the consensus-relevant information content. Computers \& Operations Research 23, 2735 .

Dong, Y., Zhang, G., Hong, W.-C., Xu, Y., 2010. Consensus models for AHP group decision making under row geometric mean prioritization method. Decision Support Systems 49, 281-289.

Herrera, F., Herrera-Viedma, E., verdegay, J. L., 1996. A model of consensus in group decision making under linguistic assessments. Fuzzy Sets and Systems 78, 73-87.

$\mathrm{Wu}, \mathrm{Z}$., Xu, J., 2012. A consistency and consensus based decision support model for group decision making with multiplicative preference relations. Decision Support Systems 52, 757-767. 